\title{
Effects of Fibroblasts Coupling on the Electrophysiology of Cardiomyocytes from Different Regions of the Human Atrium: A Simulation Study
}

\author{
Gunnar Seemann ${ }^{1,2}$, Axel Loewe ${ }^{3}$, Eike M. Wülfers ${ }^{1,2}$ \\ ${ }^{1}$ Institute for Experimental Cardiovascular Medicine, University Heart Center Freiburg · Bad \\ Krozingen, Medical Center - University of Freiburg, Freiburg, Germany \\ ${ }^{2}$ Medical Faculty, University of Freiburg, Freiburg, Germany \\ ${ }^{3}$ Institute of Biomedical Engineering, Karlsruhe Institute of Technology, Karlsruhe, Germany
}

\begin{abstract}
Atrial fibrillation is a common cardiac arrhythmia. The disturbance of the normal repolarization process due to heterogeneous myocyte-fibroblast coupling might play a role for this disease. We investigate this interaction in the heterogeneous atrium using a computational approach.

Human atrial myocyte computational models representing 10 different regions of the atrium were each coupled to a human atrial fibroblast model and the impact of the myocyte-fibroblast coupling on action potential measures was investigated.

Myocytes from the pulmonary vein are affected most by the coupling to fibroblasts. Action potential amplitude is reduced from $105 \mathrm{mV}$ to $94 \mathrm{mV}$ and the upstroke velocity changes from $192 \mathrm{~V} / \mathrm{s}$ to $152 \mathrm{~V} / \mathrm{s}$, potentially reducing the conduction velocity. In general, the action potential duration of myocytes with short action potentials is prolonged and that of those with long is shortened.

The large effect on pulmonary vein action potentials is mainly due to reduced $I_{K 1}$ in these cells compared to other regions of the atrium. The strong effects of fibroblast coupling to pulmonary vein myocytes are likely to be an additional reason for the crucial role of the pulmonary veins in atrial fibrillation.
\end{abstract}

\section{Introduction}

Cardiac arrhythmias are a high burden to the health systems and quality of life. The most common arrhythmia is atrial fibrillation (AF), which affects more than $10 \%$ of the population older than 70 and more than $1 \%$ of the population in general with an upward trend [1]. The mechanisms of initiation and perpetuation of this arrhythmia are not yet fully understood. Both trigger and substrate changes need to be present in order to allow progression of AF. In this regard, the pulmonary vein (PV) regions play an important role as they are often source of triggered activity. Substrate changes, e.g., by fibroblasts coupling to myocytes, disturb the normal repolarization process. This might play a significant role in the perpetuation of AF.

Cardiac myocytes from different regions of the atrium show differences in ion channel density [2]. This manifests in action potential (AP) morphology changes and in action potential duration (APD) variations. This heterogeneity of the AP seems to play an important role for the normal repolarization process leading to an overall more homogeneous repolarization as early activated cells tend to have a longer APD than late activated cells [2]. During progression of $\mathrm{AF}$, the remodeling processes involve the activation of fibroblasts and an increase in fibrosis in addition to electrophysiological remodeling. Up to now, it is not clear if the fibroblasts couple to atrial cardiomyocytes and how they influence the regionally varying APs. Additionally, it is not known if and how myocyte-fibroblast coupling contributes to the perpetuation and progression of atrial fibrillation. This work uses a single cell computational approach to investigates the effects of coupled fibroblasts on atrial cells from different regions of the atrium before electrophysiological remodeling happens.

\section{Materials and methods}

Atrial myocytes were represented with the human atrial and electrophysiological model of Courtemanche, Ramirez and Nattel (CRN) [3]. In order to describe cells from different regions of the atrium, the variations of the healthy, non-remodeled CRN model proposed by Krueger et al. were used [2]. The 10 different regions are the right and left atrial working myocardium (RA and LA), the right and left atrial appendage (RAA and LAA), Bachmann's bundle (BB), the crista terminalis (CT), pulmonary veins $(\mathrm{PV})$, the septum (Sep), and the mitral and tricuspid valve ring (MVR and TVR). Each of these model variants were coupled to the human atrial fibroblast model proposed by Maleckar et al. [4]. The fibroblast model of type Active 1 

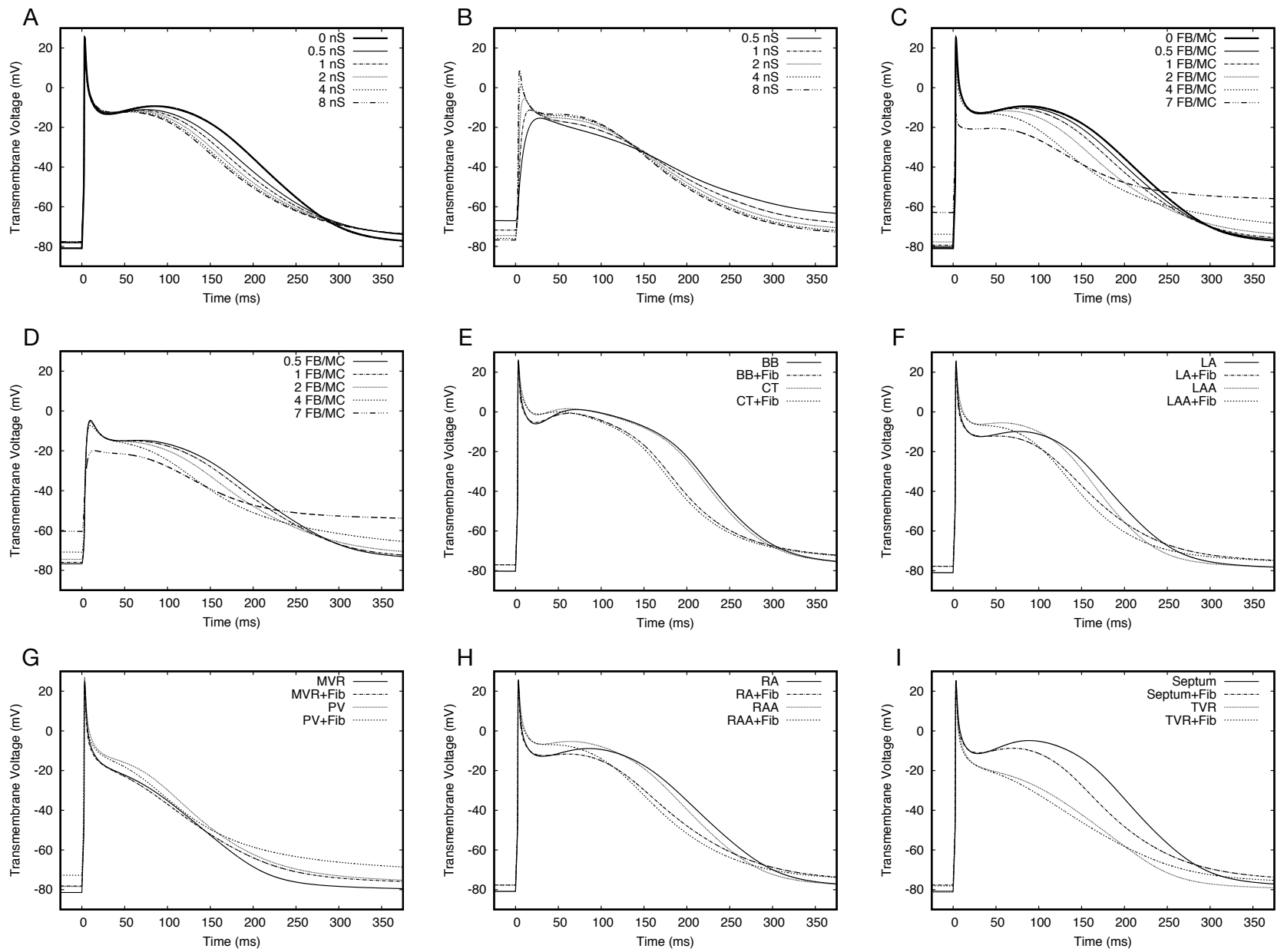

Figure 1. Effects of fibroblasts on atrial myocytes. $(\mathrm{A}+\mathrm{B})$ Varying conductivity between one myocyte and two fibroblasts with (A) the action potential of the myocyte and (B) the one of the fibroblasts. The conductivity of $0 \mathrm{nS}$ indicates the regular RA model without fibroblast coupling. (C+D) Varying numbers of fibroblasts attached to myocytes with a constant conductivity of $2 \mathrm{nS}$ with (C) the action potential of the myocyte ans (D) the one of the fibroblasts. The coupling of $0 \mathrm{FB} / \mathrm{MC}$ (fibroblasts per myocyte) indicates the regular RA model without fibroblast coupling. (E-I) Action potential of cells from different regions of the human atrium with (+Fib) and without fibroblasts. The cells are coupled to two fibroblasts with the conductivity of $2 \mathrm{nS}$ each. BB: Bachmann's bundle; CT: crista terminalis; LA: left atrium; LAA: left atrial appendage; MVR: mitral valve ring; PV: pulmonary vein; RA: right atrium; RAA: right atrial appendage; TVR: tricuspid valve ring.

was chosen with a resting membrane voltage of $-47.8 \mathrm{mV}$. In order to avoid drifting of intracellular ion concentrations in both models, the stabilization approach proposed by van Oosterom and Jacquemet [5] was applied with a value of $\alpha=10^{-5}$. If not specified, two fibroblasts were coupled to the $\mathrm{CRN}$ model variants with a coupling conductivity of $2 \mathrm{nS}$ each for the simulations. Variations in coupling conductivity and number of fibroblasts per myocyte were adopted from Jacquemet et al. [6].

The models were solved using the Rush-Larsen method for the gating variables and the forward Euler method for all other ordinary differential equations with a time step of $10 \mu \mathrm{s}$. For atrial myocytes coupled to fibroblasts (referred to as + Fib) and uncoupled, we analyzed the following AP measures: resting membrane voltage (RMV), action potential amplitude (APA), maximum upstroke velocity $\left(\mathrm{dV} / \mathrm{dt}_{\max }\right)$, and the action potential duration at $50 \%$ and $90 \%$ repolarization $\left(\mathrm{APD}_{50}\right.$ and $\mathrm{APD}_{90}$ ). Mostly, data are presented as mean \pm standard deviation.

\section{Results}

As the number of fibroblasts coupled to myocytes and also the conductivity between myocytes and fibroblasts 
Table 1. Features of atrial myocytes of 10 different regions uncoupled or coupled (+Fib) to two fibroblasts with a conductivity of $2 \mathrm{nS}$ each. RMV: Resting membrane voltage; APA: action potential amplitude; dV/dt: maximum transmembrane voltage upstroke velocity; $\mathrm{APD}_{50 / 90}$ : action potential duration at 50/90\% repolarization.

\begin{tabular}{lrrrrrrrrrr}
\hline Feature & BB & CT & LA & LAA & MVR & PV & RA & RAA & Septum & TVR \\
\hline \hline RMV (mV) & -80.28 & -80.28 & -81.01 & -80.97 & -81.43 & -78.43 & -80.88 & -80.82 & -80.81 & -81.35 \\
RMV+Fib (mV) & -77.08 & -77.09 & -77.82 & -77.77 & -78.18 & -72.75 & -77.71 & -77.65 & -77.66 & -78.11 \\
$\Delta \mathrm{RMV} \mathrm{( \% )}$ & 3.99 & 3.97 & 3.94 & 3.95 & 3.99 & 7.24 & 3.92 & 3.92 & 3.90 & 3.98 \\
\hline $\mathrm{APA}(\mathrm{mV})$ & 106.18 & 106.37 & 106.30 & 106.49 & 106.38 & 105.05 & 106.26 & 106.40 & 106.23 & 106.35 \\
$\mathrm{APA}+\mathrm{Fib}(\mathrm{mV})$ & 101.72 & 101.88 & 102.21 & 102.30 & 102.37 & 94.21 & 102.11 & 102.17 & 102.06 & 102.33 \\
$\Delta \mathrm{APA}(\%)$ & 4.20 & 4.22 & 3.85 & 3.93 & 3.77 & 10.32 & 3.91 & 3.98 & 3.93 & 3.78 \\
\hline $\mathrm{dV}_{\mathrm{dd}}$ max $(\mathrm{V} / \mathrm{s})$ & 195.76 & 195.53 & 197.09 & 198.44 & 193.63 & 192.88 & 200.77 & 201.42 & 201.58 & 189.63 \\
$\mathrm{dV}_{\mathrm{dt}_{\text {max }}+\mathrm{Fib}(\mathrm{V} / \mathrm{s})}$ & 188.07 & 188.11 & 177.14 & 176.03 & 190.30 & 152.29 & 177.64 & 180.34 & 179.57 & 188.60 \\
$\Delta \mathrm{dV} / \mathrm{dt}_{\text {max }}(\%)$ & 3.93 & 3.79 & 10.12 & 11.29 & 1.72 & 21.04 & 11.52 & 10.47 & 10.92 & 0.54 \\
\hline $\mathrm{APD}_{50}(\mathrm{~ms})$ & 206.80 & 202.70 & 155.21 & 145.31 & 73.84 & 86.75 & 178.79 & 170.16 & 183.73 & 95.46 \\
$\mathrm{APD}_{50}+\mathrm{Fib}(\mathrm{ms})$ & 167.92 & 162.96 & 123.09 & 118.12 & 64.04 & 73.70 & 138.95 & 133.74 & 145.66 & 76.38 \\
$\Delta \mathrm{APD}_{50}(\%)$ & 18.80 & 19.61 & 20.69 & 18.71 & 13.27 & 15.04 & 22.28 & 21.40 & 20.72 & 19.99 \\
\hline $\mathrm{APD}_{90}(\mathrm{~ms})$ & 312.80 & 307.75 & 261.39 & 242.41 & 209.33 & 234.28 & 300.85 & 286.86 & 293.92 & 245.11 \\
$\mathrm{APD}_{90}+\mathrm{Fib}(\mathrm{ms})$ & 292.69 & 286.28 & 254.50 & 234.05 & 223.28 & 249.22 & 288.95 & 273.50 & 281.93 & 253.07 \\
$\Delta \mathrm{APD}_{90}(\%)$ & 6.43 & 6.98 & 2.64 & 3.45 & -6.66 & -6.38 & 3.96 & 4.66 & 4.08 & -3.25 \\
\hline
\end{tabular}

is not exactly known, we first analyzed the effect of different coupling conductivity between one myocyte and two fibroblasts (Fig. $1 \mathrm{~A}+\mathrm{B}$ ) and different numbers of fibroblast coupled to myocytes with a conductivity of $2 \mathrm{nS}$ (Fig. $1 \mathrm{C}+\mathrm{D}$ ) on the AP of the normal CRN model and the fibroblast model. The influence of varying the conductivity (from $0 \mathrm{~ns}$ to $8 \mathrm{nS}$ ) of the myocyte shows an almost conductivity independent less negative RMV and a shortening of the $\mathrm{APD}_{50}$ (Fig. $1 \mathrm{~A}$ ) on the AP. The AP of the fibroblasts show a dependence of the RMV, $\mathrm{dV} / \mathrm{dt}_{\max }$, and $\mathrm{APD}_{90}$, but not of $\mathrm{APD}_{50}$ on conductivity changes (Fig. $1 \mathrm{~B}$ ). The influence of varying the number of fibroblasts coupled to each myocytes (from $0.5 \mathrm{FB} / \mathrm{MC}$ to $7 \mathrm{FB} / \mathrm{MC}$ ) on the AP of the myocyte shows an effect on the RMV and the $\mathrm{APD}_{50}$ and less on $\mathrm{APD}_{90}$ (Fig. $1 \mathrm{C}$ ). When coupling $8 \mathrm{FB} / \mathrm{MC}$, the AP of the myocyte was unphysiological. The AP of the fibroblasts shows little dependence of $\mathrm{dV} / \mathrm{dt}_{\text {max }}$ and $\mathrm{APD}_{90}$ on the number of fibroblasts per myocyte, but $\mathrm{APD}_{50}$ is impacted. As $2 \mathrm{nS}$ and $2 \mathrm{FB} / \mathrm{MC}$ show a medium influence on the AP morphology, we decided to use these values to investigate the impact of fibroblasts on atrial myocytes of different regions of the atrium shown in Figure 1 E-I. The influence on the heterogeneous de- and repolarization measures is shown in Table 1.

Figure 1 E-I show that all myocytes from different regions of the atrium have a less negative RMV by $4.28 \%$ (standard deviation: 1.04\%) when coupled to fibroblasts. The largest difference can be seen for PV cells (Fig. 1 G) where the RMV changed by $7.24 \%$. The smallest influence was seen for the septum. Also, APA is reduced for all myocytes by $4.59 \%(2.02 \%)$. Again, the strongest impact was seen in PV cells with a reduction from $105 \mathrm{mV}$ to $94 \mathrm{mV}$. Here, the smallest impact was seen for the MVR. The maximum upstroke velocity $\left(\mathrm{dV} / \mathrm{dt}_{\max }\right)$ is one factor influencing the conduction velocity (CV) in tissue. $\mathrm{dV} / \mathrm{dt}_{\max }$ was reduced by $8.54 \%(6.14 \%)$. Small changes in this parameter were found for $\mathrm{BB}$ and $\mathrm{CT}$ as conducting structures as well as MVR and especially TVR. The PV cells were again influenced the most with a reduction of $\mathrm{dV} / \mathrm{dt}_{\max }$ from $193 \mathrm{~V} / \mathrm{s}$ to $152 \mathrm{~V} / \mathrm{s}$.

The strongest effect of myocyte-fibroblast coupling was seen for $\mathrm{APD}_{50}$ with a reduction by $19.05 \%$ (2.83\%) leading to a faster initial repolarization in each myocyte. The strongest influence was on RA cells (179 ms to $138 \mathrm{~ms}$ ) and the smallest on MVR (74 ms to $64 \mathrm{~ms}$ ). Final repolarization is characterized by $\mathrm{APD}_{90}$. $\mathrm{APD}_{90}$ was affected dependent on its value in the uncoupled situation by $4.85 \%(1.62 \%)$. The APD of myocytes with short APs is prolonged and that of those with long is shortened. The $\mathrm{APD}_{90}$ of, e.g., CT is shortened from $308 \mathrm{~ms}$ to $286 \mathrm{~ms}$ whereas the $\mathrm{APD}_{90}$ of MVR is prolonged from $209 \mathrm{~ms}$ to $223 \mathrm{~ms}$.

\section{Discussion}

The simulation results demonstrate that cells from different regions of the atrium are likely to be effected dif- 
ferently by coupled fibroblasts. As longer APDs shortened and shorter prolonged, the gradient in heterogeneity of APD across the atrium is reduced. Hence, the repolarization process is less homogeneous and therefore may lead to easier induction of arrhythmia due to spatial differences in refractoriness.

The maximum upstroke velocity as a surrogate measure for $\mathrm{CV}$ was reduced the most for PV and only little for $\mathrm{BB}$ and $\mathrm{CT}$. As BB and CT are the fast conducting bundles in the atrium, the overall activation sequence might be only marginally influenced by the coupling of fibroblasts to myocytes in early AF. On the other hand, the CV in the PVs will probably be reduced, allowing reentry with shorter wavelengths. The strong effects of fibroblast coupling to PV myocytes are likely to be an additional reason for the crucial role of the PVs in AF. The reason for the larger impact of PV compared to other regions of the atrium is the less pronounced $I_{K 1}$ in the PVs leading to the less negative RMV. By this, the sodium channel availability is reduced and the maximum upstroke velocity is smaller. During electrophysiological remodeling of $\mathrm{AF}$, $I_{K 1}$ density in the cells is increasing. This might be a cellular mechanism to counteract the depolarizing influence of fibroblasts on the RMV.

In a future study, we are aiming to investigate the influence of fibroblasts on chronically remodeled atrial myocytes. Additionally, other cellular models will be considered in order to understand the model dependency. Also, different fibroblast models (e.g., [7] [8]) need to be investigated. Initial tests have shown that the model by Morgan et al. [7] uniquely prolong the $\mathrm{APD}_{90}$ of the myocyte models from all ten regions and the one by Koivumäki and colleagues [8] always shortens $\mathrm{APD}_{90}$.

Other publications like [4], [8], and [7] have also investigated the influence of myocyte-fibroblast coupling in the human atrium. Here, we consider for the first time the differential effects on a heterogeneous set of cellular models representing different regions within the human atria. These differences will need further investigations on tissue level (e.g., rate dependence of $\mathrm{CV}$ and effective refractory period and potentially a quantification of the arrhythmic risk like proposed in [9]) and ECG level (P-wave changes).

As the number of fibroblasts coupled to myocytes and the coupling conductivity is not exactly known, this work illustrates an approximation of the effects. Further experimental data is necessary to investigate this effect in more detail. Especially since not only the atrium is expected to be heterogeneous but also the amount of fibroblast in these regions. Our results suggest that the differential effects of fibroblast coupling to the cellularly heterogeneous atrial substrate are an important factor to understand AF initiation and perpetuation and underline the crucial role of the PVs.

\section{Acknowledgements}

This project was financially supported by the European Research Council Advanced Grant CardioNECT and by the German Research Foundation (SE 1758/3-3, DO 637/22-3, and CRC 1173).

\section{References}

[1] Nattel S. New ideas about atrial fibrillation 50 years on. Nature 2002;415:219-226.

[2] Krueger MW, Dorn A, Keller DUJ, Holmqvist F, Carlson J, Platonov PG, Rhode KS, Razavi R, Seemann G, Dössel O. In-silico modeling of atrial repolarization in normal and atrial fibrillation remodeled state. Med Biol Eng Comp 2013; 51(10):1105-1119.

[3] Courtemanche M, Ramirez R, Nattel S. Ionic mechanisms underlying human atrial action potential properties: Insights from a mathematical model. Am J Physiol 1998;275:H301$\mathrm{H} 321$.

[4] Maleckar MM, Greenstein JL, Giles WR, Trayanova NA. Electrotonic coupling between human atrial myocytes and fibroblasts alters myocyte excitability and repolarization. Biophys J 2009;97(8):2179-2190.

[5] van Oosterom A, Jacquemet V. Ensuring stability in models of atrial kinetics. In Proc. Comp. Cardiol., volume 2009. 2009; 69-72.

[6] Jacquemet V, Henriquez CS. Loading effect of fibroblastmyocyte coupling on resting potential, impulse propagation, and repolarization: insights from a microstructure model. Am J Physiol 2008;294(5):H2040-52.

[7] Morgan R, Colman M, Chubb H, Seemann G, Aslanidi O. Slow conduction in the border zones of patchy fibrosis stabilizes the drivers for atrial fibrillation: Insights from multiscale human atrial modeling. Front Physiol 2016;7:474.

[8] Koivumki JT, Clark RB, Belke D, Kondo C, Fedak PWM, Maleckar MMC, Giles WR. Na+ current expression in human atrial myofibroblasts: identity and functional roles. Front Physiol 2016;5:275.

[9] Loewe A, Lutz Y, Wilhelms M, Sinnecker D, Barthel P, Scholz EP, Dossel O, Schmidt G, Seemann G. Insilico assessment of the dynamic effects of amiodarone and dronedarone on human atrial patho-electrophysiology. Europace 2014;16(suppl 4):iv30-iv38.

Address for correspondence:

Dr. Gunnar Seemann

Institute for Experimental Cardiovascular Medicine

University Heart Center Freiburg · Bad Krozingen

Elsaesser Strasse 2Q, 79110 Freiburg, Germany

Gunnar.Seemann@universitaets-herzzentrum.de 